\title{
How to implement a selective colorimetric gas sensor with off the shelf components?
}

\author{
Christian Driau ${ }^{1,2}$, Cristian Fàbrega ${ }^{1,2,{ }^{*}}$, Ismael Benito-Altamirano ${ }^{1,2}$, Peter Pfeiffer $^{1,2}$, \\ Olga Casals ${ }^{1,2}$, Hongqiang Li ${ }^{3}$, Joan Daniel Prades ${ }^{1,2}$ \\ ${ }^{1}$ MIND, Departament of Electronics and Biomedical Engineering, Universitat de Barcelona (UB), Barcelona, Spain. \\ ${ }^{2}$ Institute of Nanoscience and Nanotechnology (IN²UB), Universitat de Barcelona (UB), Barcelona, Spain. \\ ${ }^{3}$ Tianjin Key Laboratory of Optoelectronic Detection Technology and Systems, School of Electronics and Information Engi- \\ neering, Tianjin Polytechnic University, Tianjin, China.
}

ABSTRACT: We report on how an inexpensive and very selective gas sensor can be implemented, simply combining colorimetric indicators casted on top of Scotch ${ }^{\circledR}$ tape, with a commercial microchip adapted here to measure optical reflectance. The system can be easily reproduced (leading to quantitatively consistent results), refreshed and reconfigured to sense different target gases, just replacing the colorimetric.

Colorimetric methods put at our disposal a wide arsenal of compounds and reaction mechanisms to address specific gaseous molecules. A colorimetric indicator is a substance that develops light absorption at specific wavelengths in the presence of the target substances. These techniques are widely spread in analytical chemistry and offer unbeatable levels of selectivity and specificity towards the target species[1-6], if compared to other popular transduction methods like solid state conductometric sensors or electrochemical devices[7-13]. However, colorimetry often raises concerns about the long-term stability of the indicator reagents and thus about the robustness of this methodology outside of a laboratory environment.

Regarding their readout, the changes developed in the light absorption spectra of the indicators can be thoroughly investigated by means of spectrophotometers. These systems are, however, bulky and practicable only for research purposes or high-end applications. To carry out measurements in the field, manual readout is also very common. In this case, an operator compares with the naked eye the color observed in the indicator with a set of references colors in a chart. This is the most simple and cheap approach, but bothersome and prone to human error. Alternatively, camera-based methods offer the chance of monitoring color changes automatically with the information available on a picture[14,15]. The challenge is avoiding external influences on the color appearance due to, e.g. ambient light or camera setup. The approach is convenient for sporadic measurements but leads to relatively costly and bulky implementations if continuous monitoring is required. In order to achieve a continuous readout in compact form factors, many research efforts have and are being devoted to 


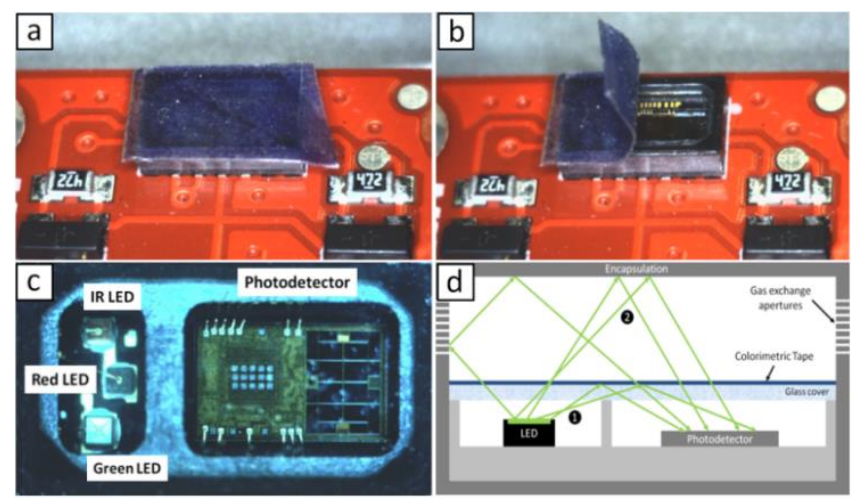

Figure 1. (a) and (b) Optical images of the MAX30105 component covered with a colorimetric tape. (c) A detailed image of the sensor chip with the red, green and IR LEDs and the photodetector. (d) Scheme of the proposed configuration: the photodetector will measure $\mathbf{0}$ the reflected light and $\mathbf{2}$ the transmitted light through the colorimetric tape (i.e. absorbance of the color indicator).

developing miniaturized systems that measure changes in absorbance/reflectance/transmittance of color indicators at specific spectral ranges[16,17]. Even though many different configurations have been proposed, all of them are based on confronting a narrow-spectrum light source (e.g. LEDs or broader emitters with filters) with a photodetector. To maximize their sensitivity to small color changes, and thus to small gas concentrations, different strategies to enlarge the optical path from the emitter to the detector through the indicator are being used[18-20]. Anyhow, the integration of the different components, especially concerning their optical alignment, is still challenging. Also, the efforts in miniaturization complicate refreshing the indicator substance if degradation occurs. In summary, the continuous readout of colorimetric indicators still raises a set of inconveniences.

In this work, we propose a simple approach to colorimetric detection of gaseous substances that combines the following advantages: 1 ) it is based on easily accessible, off the shelf commercial components, 2 ) it is compatible with a wide range of colorimetric indicators, operating at different wavelengths, 3) it is easily resettable/refreshable and 4) offers an excellent repeatability among devices.

We base our readout approach on a commercial component, the MAX30105, a "High-Sensitivity Particle-Sensing Module" from MAXIM. This integrated microsystem encloses a set of 3 internal LEDs - operating in the green (537 $\pm 35 \mathrm{~nm}$ ), the red $(660 \pm 20 \mathrm{~nm})$, and the infrared $(880 \pm 30 \mathrm{~nm}$ ) ranges -, a broad band photodiode (from 640 to $980 \mathrm{~nm},>30 \%$ Quantum Efficiency), and the corresponding control, driving, acquisition and communication modules. The device itself was conceived to address dissimilar applications like smoke particle detection in air and blood pulse oximetry monitoring; and operates sequentially measuring the amount of light reflected at different spectral ranges (i.e. green, red, infrared). This device has a footprint of $5.6 \times 3.3 \mathrm{~mm}^{2}$ and can be found in the market at a retail price of less than $4 \$$ per unit in bulk amounts. Figure 1 shows images of the device, the proposed configuration and a cross section of its internal parts.

Sensitivity to gases was incorporated by means of different colorimetric indicators. For illustration purposes we choose two different $\mathrm{pH}$-based indicators, Bromophenol Blue and $\mathrm{m}$-Cresol Purple, targeting ammonia $\left(\mathrm{NH}_{3}\right)$ and carbon dioxide $\left(\mathrm{CO}_{2}\right)$, respectively. These indicators have become a standard in the industry of colorimetric sensors as in the case of the socalled Dräger gas tubes. The indicator $(5 \mathrm{~g})$ for $\mathrm{NH}_{3}$ consisted of $0.075 \mathrm{~g}$ of Ethylcelullose and $44 \mathrm{~g}$ of Bromophenol Blue in Ethanol. The indicator $(5 \mathrm{~g})$ for $\mathrm{CO}_{2}$ consisted of $0.356 \mathrm{~mL}$ of $1 \mathrm{M}$ Tetrabutylammonium hydroxide (TBAH) in Methanol and $0.075 \mathrm{~g}$ of Ethylcelullose and $44 \mathrm{mg}$ of $\mathrm{m}$-Cresol Purple in ethanol. In dry synthetic air (SA), right after the synthesis, the $\mathrm{NH}_{3}-$ sensitive indicator was bright-yellow and the $\mathrm{CO}_{2}$-sensitive one was dark blue. In turn, in the presence of $\mathrm{NH}_{3}$ and $\mathrm{CO}_{2}$ their 


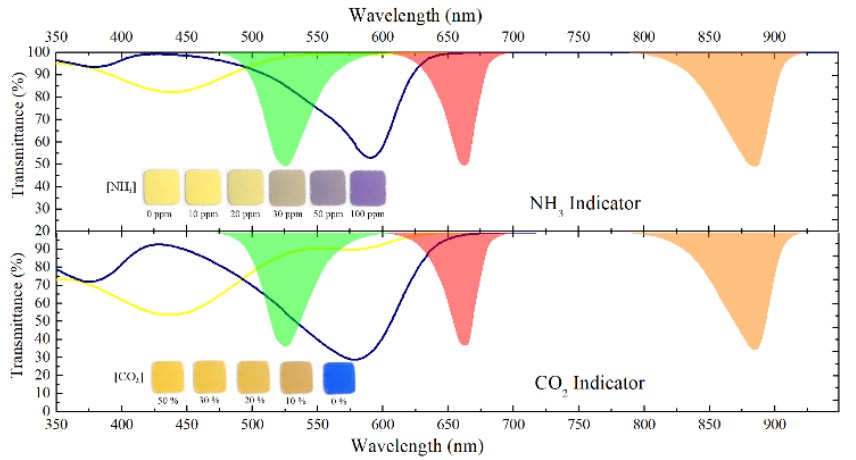

Figure 2. UV-vis transmission spectra of both $\mathrm{NH}_{3}$ and $\mathrm{CO}_{2}$ colorimetric indicators together with the emission spectra of the LEDs (Green line, Red line and Infrared line). Yellow and blue lines indicate the acid and basic state of the color indicator, respectively. A set of images of the colorimetric tapes at different concentrations of $\mathrm{CO}_{2}$ and $\mathrm{NH}_{3}$ are also depicted.

respective color shades turned to blue and yellow, in a reversible manner when exposed back to SA. Figure 2 shows the reflectance spectra of both indicators in SA and in the presence of their respective target gases.

In order to integrate the indicators on the readout chip, we spin coated each indicator on conventional Scotch ${ }^{\circledR}$ Magic ${ }^{\mathrm{TM}}$ tape (rotation at $500 \mathrm{rpm}$ for 10 seconds followed by $2000 \mathrm{rpm}$ for 20 seconds) and left them to air dry. To facilitate the handling during the spinning process, the tape was adhered on top of a glass coverslip, from where it could be easily removed after deposition. We obtained uniform indicator films spread on top of the adhesive tape. Then, the indicator-carrying pieces of tape (from hereafter "colorimetric tape") were transferred to the readout MAX30105 chip by attaching the tape directly on the chip glass surface (Figure 1). Finally, the colorimetric tape was cut to shape following the edges of the chip with a blade. This process was repeated to produce newly refreshed devices with excellent reproducibility. Also, the methodology made it possible changing the functionality of the sensor, just replacing the colorimetric tape (as a replaceable sticker) and reusing the readout chip.

To investigate the response to gases of the here-described sensors, we placed them into a gas tight dark chamber coupled to a gas mixing system feeding a flow of $200 \mathrm{ml} / \mathrm{min}$ of different gas blends. The sensors were read externally with an ESP8266 microcontroller through a ${ }^{2} \mathrm{C}$ bus fed through the chamber walls. The internal logic of the MAX30105 allows for many different settings, like different integration times, acquisition range and number of samples averaged in the photodiode; as well as different light intensities emitted by the LEDs. In our experiments, the optimum conditions were found to be an integration time of $411 \mu \mathrm{S}$, a least significant bit of $31.25 \mathrm{pA}$ and 16 samples averaged per measurement, without much impact of these parameters on the here-reported results. The role of the LED intensity will be discussed in detail later.

Figure 3 shows a summary of the responses to $\mathrm{NH}_{3}$ and $\mathrm{CO}_{2}$ obtained with the here-proposed sensor system. Clear responses in the red and in the green channels were observed, but none in the infrared as expected from the matching between the emission spectrum of the LEDs and the absorption bands of the colorimetric indicator (see Figure 2). For simplicity, and since the response signal in the red and green channels were similar, we will use the only the green LED data in the forthcoming discussion. 

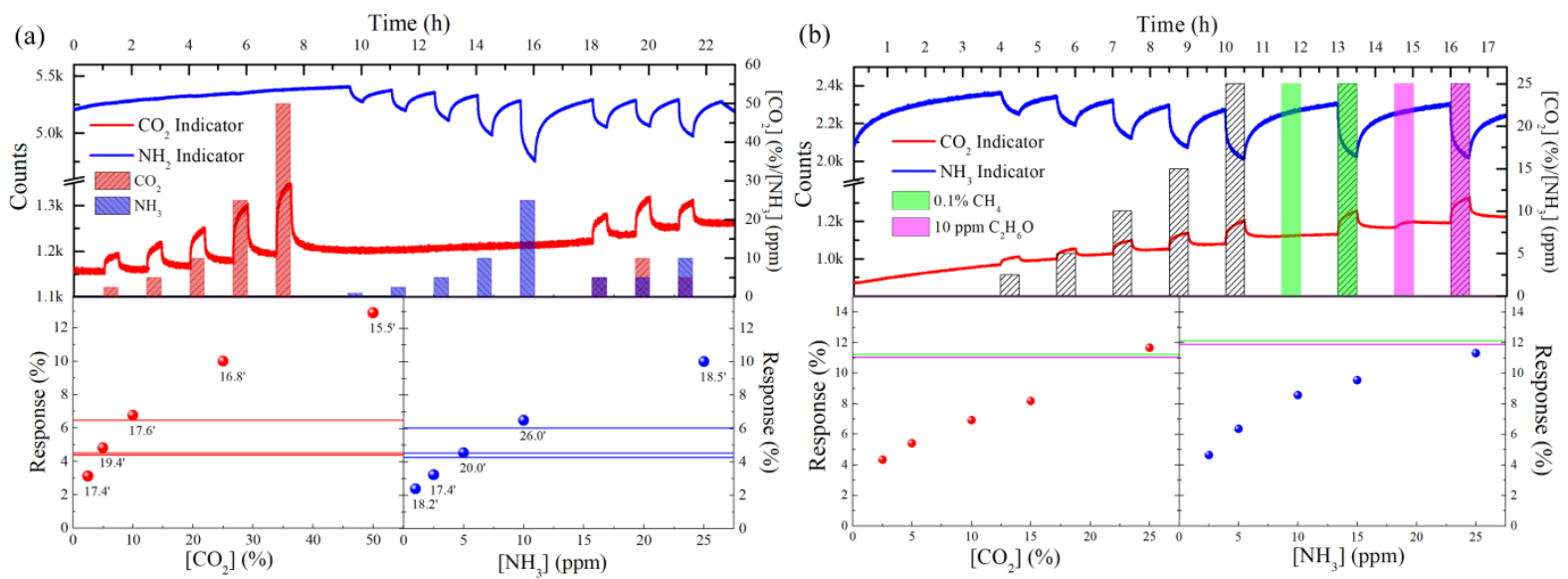

Figure 3. Dynamic response of the $\mathrm{NH}_{3}$ (blue line) and $\mathrm{CO}_{2}$ (red line) colorimetric sensors under different concentrations and cross-interference experiments (a) between them (b) and with methane $\left(\mathrm{CH}_{4}\right)$ and ethanol $\left(\mathrm{C}_{2} \mathrm{H}_{6} \mathrm{O}\right)$. Lower panels show the response values and times for both sensors (circles and minutes) together with the responses obtained in the cross-interference experiments (horizontal lines). Data acquired with the green LED operating at $41 \mathrm{~mW}$.

On the one hand, $\mathrm{NH}_{3}$ concentrations ranging from $2.5 \mathrm{ppm}$ to $25 \mathrm{ppm}$ were measured, with responses up to $10 \%{ }^{1}$. On the other hand, the $\mathrm{CO}_{2}$ concentrations investigated ranged from $2.5 \%$ to $50 \%$, with responses up to $12 \%$. The response and recovery times were around 20 minutes in all cases. It may perhaps be observed without straying too far afield from our primary focus that the response can be improved by adjusting the formulation of the colorimetric inks. For example, just by diluting the original formulation response times in the sub-minute range can be achieved (see S.I.)

To investigate the selectivity of the here-proposed sensors, both devices were exposed to mixtures containing $\mathrm{NH}_{3}$ and $\mathrm{CO}_{2}$ simultaneously (see Figure 3a). Clearly, each indicator responded exclusively to its target gas, without being affected by the other one (no cross-interference). Similar interference experiments were carried out with other gases (ethanol, and $\mathrm{CH}_{4}$ ) leading to identical conclusions (see Figure $3 b$ ).

Concerning the power consumption of the MAX30105 system, it is mostly determined by the power needed to lit on the LEDs: while the control, driving and communication electronics consume less than $1 \mathrm{~mW}$ during the acquisition, the electrical power dissipated at the LEDs can be varied widely - from $0.65 \mathrm{~mW}$ to $165 \mathrm{~mW}$ (green LED) - depending on the light emission set point. Evidently, higher light intensities provide larger absolute signals, while the absolute noise level remains approximately constant. Accordingly, the relative noise level is higher at lower light intensities (see Figure 4). We have also observed that the light intensity does not affect significantly the response to gases, which, according to our definition, it is a relative change of the signal. Therefore, light intensity only determines the gas detection limit; this is the point at which the response

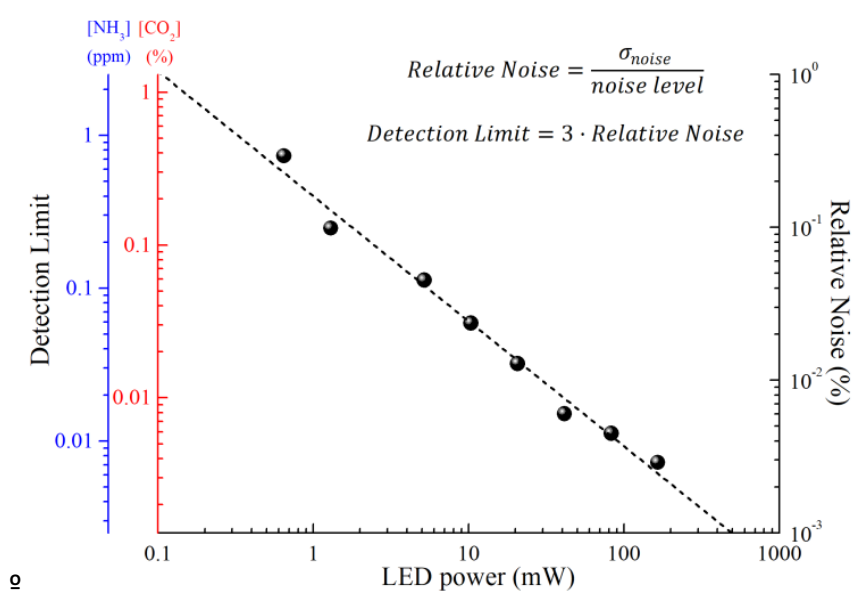

Figure 4. The theoretical detection limits for the $\mathrm{NH}_{3}$ and $\mathrm{CO}_{2}$ colorimetric tapes (left axis) and relative noise levels (right axis) at different LED power settings. 


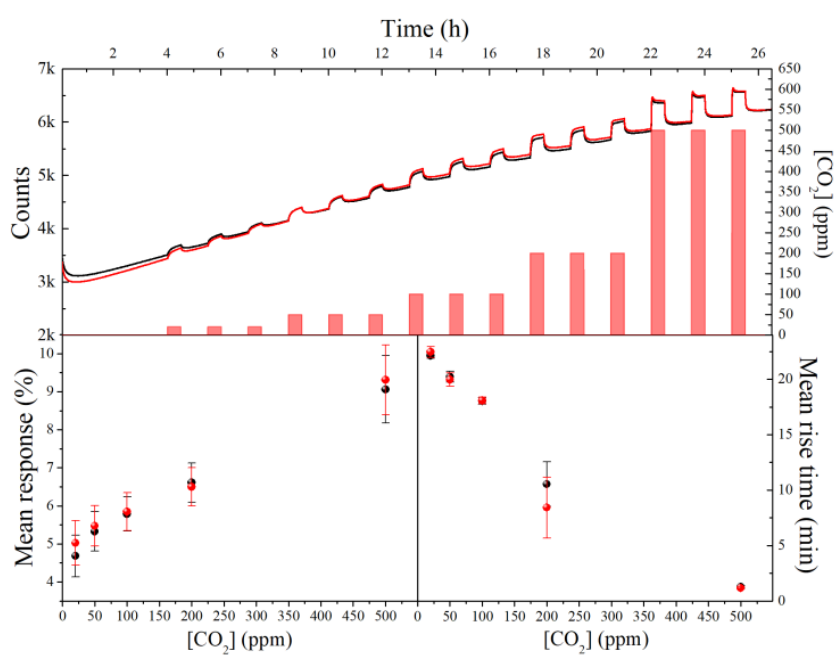

Figure 5. Dynamic responses towards $\mathrm{CO}_{2}$ in the range of environmental applications for two identical tapes (top). Summary of averaged responses and rise time of each concentration. In both cases, the green LED data was considered.

is comparable to the relative noise level. Figure 5 shows the detection limits measured at different LED powers (i.e. light intensities). Clearly, the detection limit can be lowered increasing the power consumption. As a matter of fact, and without any further optimization, the proposed setup can achieve detection limits in the range of 1 ppb for $\mathrm{NH}_{3}$ and $200 \mathrm{ppm}$ for $\mathrm{CO}_{2}$ operating the LEDs at the maximum power $(165 \mathrm{~mW})$. If a minimum power consumption were required (1 mW for the electronics plus $0.65 \mathrm{~mW}$ for the LED), detection limits would raise to $1 \mathrm{ppm}$ for $\mathrm{NH}_{3}$ and $1 \%$ for $\mathrm{CO}_{2}$.

It is worth insisting on the fact that the gas sensor behavior of our devices is mostly determined by the colorimetric indicator. To illustrate this, we modified the formulation of the $\mathrm{CO}_{2}$ indicator to measure much lower concentrations, which are of interest in environmental applications (up to $500 \mathrm{ppm}$ ). To that end, the $\mathrm{pH}$ of the fresh indicator was shifted towards the inflection point of the titration curve[21] by modifying the amount of TBAH $(0.285 \mathrm{~mL}$ in $5 \mathrm{~g}$ of indicator) of the original formula. Figure 5 shows the response to $\mathrm{CO}_{2}$ of two of these new colorimetric tapes applied on top of two different MAX30105 chips. Now, this new gas sensor operates in the hundreds of ppm range, with similar responses as before.

This last experiment also served to prove the repeatability of the approach. The two tracks shown in Figure 5 were nearly identical and corresponded to two different pieces of colorimetric tape on two different MAX30105 devices. Similar reproducible results were obtained routinely following our fabrication procedure, provided that colorimetric tapes produced in the same deposition batch were used. Further optimization could be achieved by optimizing the thickness of the indicator layer, playing around with the other on-chip settings, and housing the device in an enclosure to provide a stable optical environment. It is worth noting that the signals recorded display a mostly monotonous baseline drift, which is quite common in $\mathrm{pH}$-based colorimetric indicators. In contrast, no-drift was observed in long term measurements using only a piece of nonimpregnated tape as a test sample. This demonstrates that neither the tape itself or the readout device are accountable for the drifts observed. In any case, drift correction is widespread issue in chemical sensors that can be addressed using manifold signal processing techniques [22] (see S.I.).

In conclusion, we have presented a method to implement a highly specific gas sensor with an off the shelf component, the MAX30105 optical sensor, and conventional colorimetric indicator recipes. The sensor has a cost of less than $4 \$$ and can be easily replicated, with excellent reproducibility. The device operates at different wavelengths and with moderate power requirements (starting from a few $\mathrm{mW}$ ). The approach has also the advantage of being easily resettable/refreshable, as the gas sensitive color indicator layer can be easily removed and replaced by a new one. This also allows for addressing other target 
gases, as simply as just replacing the indicator, and reusing the readout chip. Therefore, the proposed principle offers a good trade of between cost, simplicity, convenience, and good gas sensing performance; circumventing some of the limitations of colorimetric indicators in long term operation.

\section{ACKNOWLEDGMENT}

The research leading to these results has received funding from the European Research Council under the European Union's Seventh Framework Program (FP/2007-2013)/ERC Grant Agreement n. 336917, the H2020 Framework Pro-gram ERC Grant Agreement n. 727297, and by the National Natural Science Foundation of China (Grant Nos. 61711530652, 61675154). J.D.Prades acknowledges the support from the Serra Húnter Program.

\section{REFERENCES}

[1] B. Kaur, N. Kaur, S. Kumar, Colorimetric metal ion sensors - A comprehensive review of the years 2011-2016, Coord. Chem. Rev. 358 (2018) 1369. doi:10.1016/J.CCR.2017.12.002.

[2] E. Priyadarshini, N. Pradhan, Gold nanoparticles as efficient sensors in colorimetric detection of toxic metal ions: A review, Sensors Actuators B Chem. 238 (2017) 888-902. doi:10.1016/J.SNB.2016.06.081.

[3] C. Pannek, K. Schmitt, J. Wollenstein, Colorimetric materials for gas selective sensing in low-power applications, in: 2015 Transducers - 201518 th Int. Conf. Solid-State Sensors, Actuators Microsystems, IEEE, 2015: pp. 1452-1455. doi:10.1109/TRANSDUCERS.2015.7181208.

[4] R. Paolesse, S. Nardis, D. Monti, M. Stefanelli, C. Di Natale, Porphyrinoids for Chemical Sensor Applications, (n.d.). doi:10.1021/acs.chemrev.6b00361.

[5] and C.-Y.L. Po-Ren Chung, Chun-Ta Tzeng, Ming-Tsun Ke, Formaldehyde Gas Sensors: A Review, Sensors. (n.d.). doi:10.3390/s130404468.

[6] C. Fabrega, L. Fernández, O. Monereo, A. Pons-Balagué, E. Xuriguera, O. Casals, A. Waag, J.D. Prades, Highly Specific and Wide Range NO2 sensor with color readout., ACS Sensors. (2017) acssensors.7b00463. doi:10.1021/acssensors.7b00463.

[7] F. Shao, J.D. Fan, F. Hernández-Ramírez, C. Fàbrega, T. Andreu, A. Cabot, J.D. Prades, N. López, F. Udrea, A. De Luca, S.Z. Ali, J.R. Morante, $\mathrm{NH}<\mathrm{inf}>3<$ /inf $>$ sensing with self-assembled ZnO-nanowire $\mu \mathrm{hP}$ sensors in isothermal and temperature-pulsed mode, Sensors Actuators, $\mathrm{B}$ Chem. 226 (2016). doi:10.1016/j.snb.2015.11.109.

[8] F. Shao, F. Hernández-Ramírez, J.D. Prades, C. Fàbrega, T. Andreu, J.R. Morante, Copper (II) oxide nanowires for p-type conductometric NH3 sensing, Appl. Surf. Sci. 311 (2014) 177-181.

J. Guilera, C. Fàbrega, O. Casals, F. Hernández-Ramírez, S. Wang, S. Mathur, F. Udrea, A. De Luca, S.Z. Ali, A. Romano-Rodríguez, J.D. Prades, J.R. Morante, Facile integration of ordered nanowires in functional devices, Sensors Actuators, B Chem. 221 (2015) 104-112. doi:10.1016/j.snb.2015.06.069.

[10] C.Fàbrega; O.Casals; F.Hernández-Ramírez; J.D.Prades, A Review on Efficient Self-Heating in Nanowire Sensors: Prospects for Very-Low Power Devices, Sensors Actuators B Chem. (2017). doi:10.1016/J.SNB.2017.10.003.

[11] J.R. Stetter*, J. Li, Amperometric Gas SensorsA Review, (2008). doi:10.1021/CR0681039.

[12] E. Bakker, Y. Qin, Electrochemical sensors., Anal. Chem. 78 (2006) 3965-84. doi:10.1021/ac060637m.

[13] T. Liu, X. Wang, L. Li, J. Yu, Review-Electrochemical NO × Gas Sensors Based on Stabilized Zirconia, J. Electrochem. Soc. 164 (2017) B610-B619. doi:10.1149/2.0501713jes.

[14] N.A. Rakow, A. Sen, M.C. Janzen, J.B. Ponder, K.S. Suslick, Molecular Recognition and Discrimination of Amines with a Colorimetric Array**, Angew. Chem. Int. Ed. 44 (2005) 4528-4532. doi:10.1002/anie.200500939.

[15] K. Schmitt, K. Tarantik, C. Pannek, I. Benito-Altamirano, O. Casals, C. Fàbrega, A. Romano-Rodríguez, J. Wöllenstein, J.D. Prades, Colorimetric sensor for bad odor detection using automated color correction, in: L. Fonseca, M. Prunnila, E. Peiner (Eds.), International Society for Optics and Photonics, 2017: p. 102461F. doi:10.1117/12.2265990.

[16] Z. Li, K.S. Suslick, Portable Optoelectronic Nose for Monitoring Meat Freshness, ACS Sensors. 1 (2016) 1330-1335. doi:10.1021/acssensors.6b00492.

[17] C. Lin, X. Xian, X. Qin, D. Wang, F. Tsow, E. Forzani, N. Tao, High Performance Colorimetric Carbon Monoxide Sensor for Continuous Personal Exposure Monitoring, ACS Sensors. 3 (2018) 327-333. doi:10.1021/acssensors.7b00722.

[18] K. Schmitt, J. Rist, C. Peter, J. Wöllenstein, Low-cost fiber-optic waveguide sensor for the colorimetric detection of ammonia, Microsyst. Technol. 
18 (2012) 843-848. doi:10.1007/s00542-011-1382-z.

[19] Y. Xiong, C.-J. Wang, T. Tao, M. Duan, S.-W. Fang, M. Zheng, A miniaturized fiber-optic colorimetric sensor for nitrite determination by coupling with a microfluidic capillary waveguide, Anal. Bioanal. Chem. 408 (2016) 3413-3423. doi:10.1007/s00216-016-9415-1.

[20] J. Courbat, D. Briand, J. Damon-Lacoste, J. Wöllenstein, N.F. de Rooij, Evaluation of pH indicator-based colorimetric films for ammonia detection using optical waveguides, Sensors Actuators B Chem. 143 (2009) 62-70. doi:10.1016/J.SNB.2009.08.049.

[21] D. Zhao, D. Miller, X. Xian, F. Tsow, E.S. Forzani, Sensors and Actuators B : Chemical A novel real-time carbon dioxide analyzer for health and environmental applications, Sensors Actuators B. Chem. 195 (2014) 171-176. doi:10.1016/j.snb.2013.12.110.

[22] S. Di Carlo, M. Falasconi, Drift Correction Methods for Gas Chemical Sensors in Artificial Olfaction Systems: Techniques and Challenges, in: W. Wang (Ed.), Adv. Chem. Sensors, IntechOpen, Rijeka, 2012. doi:10.5772/33411. 
Table of content

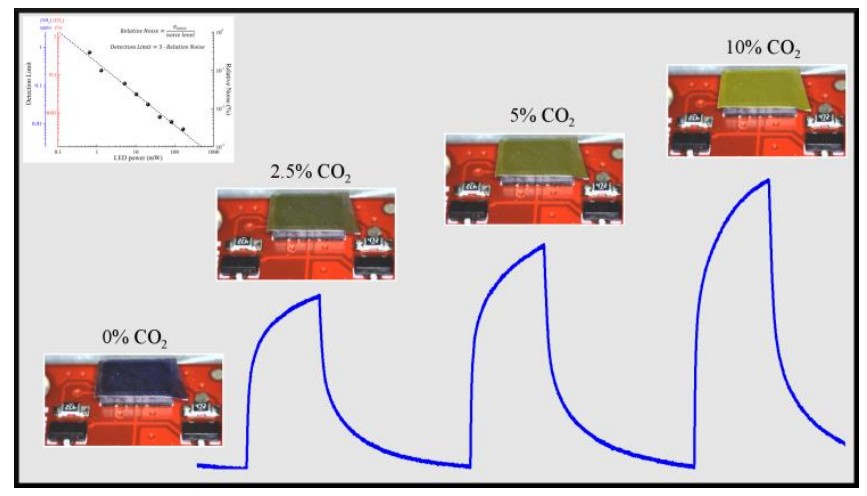




\title{
How to implement a selective colorimetric gas sensor with off the shelf components?
}

\author{
Christian Driau ${ }^{1,2}$, Cristian Fàbrega ${ }^{1,2,{ }^{*}}$, Ismael Benito-Altamirano ${ }^{1,2}$, Peter Pfeiffer ${ }^{1,2}$, \\ Olga Casals ${ }^{1,2}$, Hongqiang $\mathrm{Li}^{3}$, Joan Daniel Prades ${ }^{1,2}$ \\ ${ }^{1}$ MIND, Departament of Electronics and Biomedical Engineering, Universitat de Barcelona (UB), \\ Barcelona, Spain. \\ ${ }^{2}$ Institute of Nanoscience and Nanotechnology (IN²UB), Universitat de Barcelona (UB), Barcelona, Spain. \\ ${ }^{3}$ Tianjin Key Laboratory of Optoelectronic Detection Technology and Systems, School of Electronics and \\ Information Engineering, Tianjin Polytechnic University, Tianjin, China.
}

\section{Optimization of the colorimetric ink: Case of $\mathrm{CO}_{2}$}

The optimization of the colorimetric ink is fundamental to achieve the necessary characteristics to improve the performance of the sensors (response time, detection range, etc.).

\section{Detection Range.}

It's been already proof in the main manuscript that the detection range can be tuned in order to detect different concentration ranges of the targeted gas $\left(\mathrm{CO}_{2}\right)$. By controlling the $\mathrm{pH}$ of the fresh indicator, it is possible to shift the inflection point of the titration curve to be more sensitive in the hundreds of ppm region (environmental applications) whereas the original ink was formulated to work in the region of interest of a breath sensor $\left(10-100 \%\right.$ of $\left.\mathrm{CO}_{2}\right)$.

\section{Response Time.}

Response time is another magnitude that is critical in many applications and highly dependent on the formulation of the inks. To prove that our inks can be also tuned to be operative in a wide range of response times, the original ink was diluted with methanol. Figure S1.a shows the normalized signal of the green LED for a concentration of $20 \%$ of $\mathrm{CO}_{2}$ for the different dilutions levels. From the shape of the signal, it is clear that the response time was decreasing with the dilution level, from several minutes (13 minutes) down to barely 30 seconds (sub-minute range). 

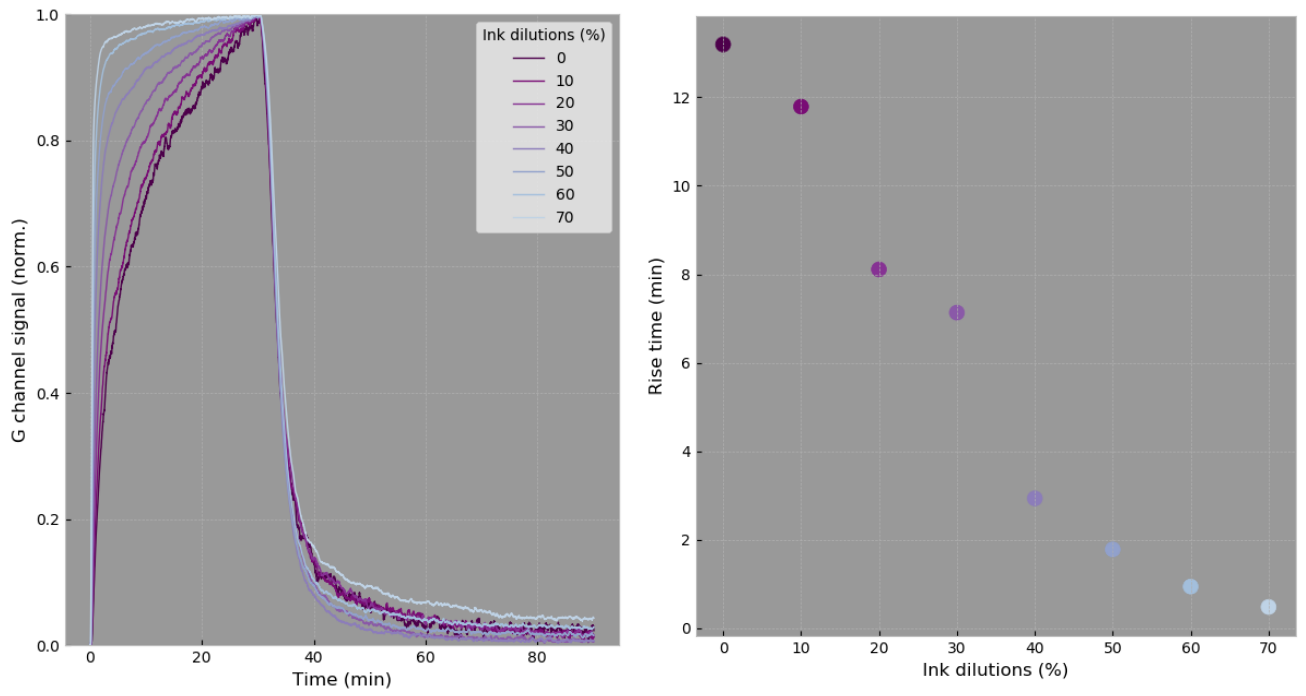

\section{Baseline Correction.}

The signal drift can be easily removed by applying simple signal processing techniques to the raw data. The following image shows how the baseline drift is completely removed (left) with a derivative/integration filter (more explicitly, taking the numerical derivative of the sensor signal, followed by a sliding window average of 5000 samples to remove the derivative offset and integrating again to recover the original signal without drift). This signal processing can be carried out in real time.
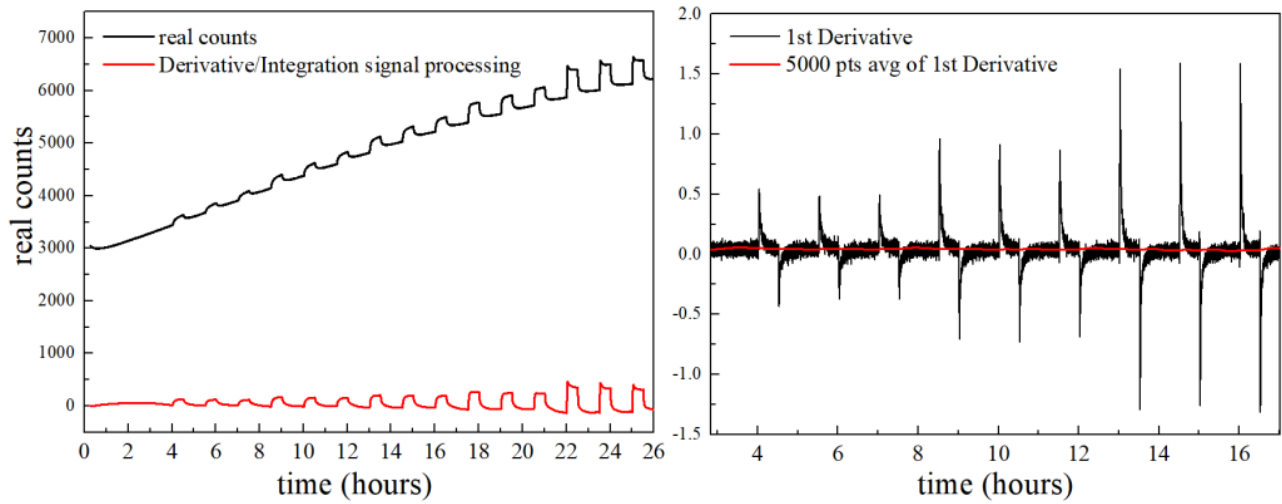

This is the simplest approach, since there exist much more complex algorithms like piece-wise orthogonal signal correction or adaptive filters to name a few that could improve further these results. 